\title{
ESPÉCIES E/OU LINHAGENS DE TRICHOGRAMMA SPP. (HYMENOPTERA: TRICHOGRAMMATIDAE) PARA O CONTROLE DE ANTICARSIA GEMMATALIS (LEPIDOPTERA: NOCTUIDAE)
}

\author{
U.R. Vianna ${ }^{1}$, D. Pratissoli ${ }^{1}$, J.C. Zanuncio ${ }^{2}$, J.R.C.C. de Alencar ${ }^{1}$, F.D. Zinger ${ }^{1}$ \\ ${ }^{1}$ Centro de Ciências Agrárias, Departamento de Produção Vegetal, Núcleo de Desenvolvimento Científico \\ e Tecnológico em Manejo Fitossanitário de Pragas e Doenças, Setor de Entomologia, CP 16, CEP 29500-000, \\ Alegre, ES, Brasil. E-mail: ulyssesvianna@hotmail.com
}

\section{RESUMO}

Trichogramma spp. (Hymenoptera: Trichogrammatidae) parasitam ovos de Anticarsia gemmatalis (Lepidoptera: Noctuidae), o principal desfolhador de soja no Brasil. O objetivo deste trabalho foi avaliar o parasitismo de onze espécies/linhagens de Trichogramma em cartelas de cartolina com vinte ovos de A. gemmatalis por fêmea desse parasitoide durante 24 horas. A linhagem de Trichogramma atopovirilia (Hymenoptera: Trichogrammatidae) de Sete Lagoas, Minas Gerais, apresentou melhor desempenho com 19 ovos de $A$. gemmatalis parasitados por fêmea em 24 horas, viabilidade de $98 \%$ e razão sexual de 0,84. A linhagem de Trichogramma pretiosum de Cristalina, Goiás, parasitou 11,9 ovos de A. gemmatalis por fêmea com $98 \%$ de viabilidade e razão sexual de 0,65. Esse trabalho confirma a importância de se avaliar linhagens de Trichogramma antes de programas de controle biológico, pois diferentes linhagens de uma mesma espécie desse parasitoide apresentam resultados distintos.

PALAVRAS-CHAVE: Glycine Max, lagarta da soja, controle biológico, parasitoide de ovos.

\begin{abstract}
TRICHOGRAMMA SPP. (HYMENOPTERA: TRICHOGRAMMATIDAE) SPECIES AND STRAINSFOR THECONTROLOF ANTICARSIA GEMMATALIS (LEPIDOPTERA:NOCTUIDAE). Several Trichogramma (Hymenoptera: Trichogrammatidae) species parasitize Anticarsia gemmatalis (Lepidoptera: Noctuidae) eggs, the major soybean desfoliator in Brazil. The objective of this work was to evaluate the parasitism of eleven Trichogramma species/strains in cardboards with twentyeggs of A. gemmatalis by this parasitoid female for 24 hours. The strain trat - 2 Trichogramma atopovirilia (Hymenoptera: Trichogrammatidae) of Sete Lagoas, Minas Gerais, showed better performance with 19 A. gemmatalis eggs parasitized by female in 24 hours, $98 \%$ viability and sex ratio of 0.84 . The strain Trichogramma pretiosum of Crystalline, Goiás, parasitized 11.9 A. gemmatalis eggs per female with $98 \%$ viability and sex ratio of 0.65 . This work confirms the importance of evaluating Trichogramma strains before biological control programs, because different strains of the same species of parasitoid have distinct results.
\end{abstract}

KEY WORDS: Glycine max, velvetbean caterpillar, biological control, egg parasitoid.

\section{INTRODUÇÃO}

Alagarta da soja Anticarsiagemmatalis (Lepidoptera: Noctuidae) é o principal desfolhador da cultura da soja na América do Norte e do Sul (MACrAE et al., 2005). Uma única lagarta pode consumir cerca de 110 $\mathrm{cm}^{2}$ de folhas de soja (WALKER et al., 2000) e aplicações expressivas de inseticidas são feitas em regiões com relatos da ocorrência desse inseto (MACRAE et al., 2005; MiKLos et al., 2007).
Inseticidas químicos, utilizados no controle de lagartas desfolhadoras de soja, provocam prejuízos financeiros, desequilibram a cadeia alimentar, elevam pragas secundárias à categoria de pragaschave, causam surgimento de novas pragas e pragas resistentes. Essas consequências estão diretamente relacionadas ao largo espectro desses produtos e às aplicações realizadas de forma intensiva e indiscriminada deles (Thomas; Boethel, 1994; Hegazi et al., 2007).

${ }^{2}$ Universidade Federal de Viçosa, Departamento de Biologia Animal/BIOAGRO, Viçosa, MG, Brasil. 
A globalização dos mercados consumidores e a exigência de alimentos de melhor qualidade têm levado ao desenvolvimento de métodos de manejo de pragas como práticas culturais para favorecer o estabelecimento de inimigos naturais (HuMmel et al., 2002; GRIESHOP et al., 2008; SHARLEY et al., 2008); ao desenvolvimento de variedades precoces ou resistentes, como a soja IAC 100, que afetam o desenvolvimento de pragas (LOURENÇÃo et al., 2000; Matos Neto et al., 2002).

Trichogramma spp. destacam-se entre os agentes biológicos, por parasitarem ovos de pragas agrícolas (Pratissoli et al., 2004a) e florestais (Oliveira et al., 2000; Oliveira et al., 2003; SOAREs et al., 2007), principalmente da ordem Lepidoptera. Esses parasitoides são utilizados em programas de controle biológico de pragas de diferentes culturas, em liberações inundativas, em cerca de 30 países (Pratissoli et al., 2002).

O sucesso de programas de controle biológico com Trichogramma spp. depende de etapas como a coleta, identificação, manutenção no laboratório e seleção de espécies e/ou linhagens do parasitoide para a praga visada (PRATISSOLI et al., 2002). O potencial de Trichogramma em soja no Brasil foi demonstrado com o relato de ocorrência de ovos de A. gemmatalis parasitados naturalmente em campo (CAÑete; Foerster, 2003). Assim, o objetivo deste trabalho foi selecionar espécies e/ou linhagens de Trichogramma com potencial de serem utilizadas para o controle de A. gemmatalis.

\section{MATERIAL E MÉTODOS}

O experimento foi realizado no Setor de Entomologia do Núcleo de Desenvolvimento Científico e Tecnológico em Manejo Fitossanitário de Pragas e Doenças (NUDEMAFI) do Centro de Ciências Agrárias da Universidade Federal do Espírito Santo (CCA-UFES) em Alegre, Espírito Santo, em câmaras climáticas a $25 \pm 1^{\circ} \mathrm{C}, 70 \pm 10 \%$ UR e fotoperíodo de 12 horas.

As espécies de parasitoides foram retiradas da coleção estoque do NUDEMAFI onde são mantidas com ovos do hospedeiro alternativo Anagasta kuehniella (Lepidoptera: Pyralidae), criado em dieta à base de farinha de trigo integral $(60 \%)$ e de milho $(37 \%)$ e levedura de cerveja (3\%).

Adultos das espécies e/ou linhagens de Trichogramma (Tabela 1) foram mantidos em recipientes de vidro $(3 \times 9 \mathrm{~cm})$ e alimentados com gotículas de mel depositados na parede interna deles.

Ovos de A. gemmatalis, obtidos da criação estoque para o processo de seleção da espécie e/ou linhagem de Trichogramma, foram acondicionados em potes plásticos de $1.100 \mathrm{~mL}$ com a tampa furada e vedada com organza para aumentar a aeração. Após o nascimento das lagartas, essas foram alimentadas com dieta artificial (GreENe et al., 1976).

Adultos de $A$. gemmatalis foram acondicionados em gaiolas de madeira $(40 \times 40 \times 40 \mathrm{~cm}) \mathrm{com}$ as laterais teladas e com tampa de vidro em salas climatizadas a $25 \pm 2^{\circ} \mathrm{C}$ e fotofase de $12 \mathrm{~h}$ e com pouca luminosidade (penumbra) na escotofase para estimular a cópula. Esses adultos foram alimentados com uma solução nutritiva (mel 10,5 g, água destilada $1,05 \mathrm{~L}$, cerveja $350 \mathrm{~mL}$, sacarose $60 \mathrm{~g}$, nipagin 1,05 g, acido ascórbico 1,05 g) embebida em chumaço de algodão em uma placa de Petri $(15 \times 1,5$ $\mathrm{cm})$ para o fornecimento de nutrientes necessários à maturação dos ovários das fêmeas e aumentar a longevidade dos adultos. As posturas foram coletadas em folhas de papel branco no interior das gaiolas, as quais foram recortadas e colocadas nos potes de criação com a dieta artificial.

Tabela 1 - Espécies e/ ou linhagens de Trichogramma (Hymenoptera: Trichogrammatidae), códigos para sua identificação e locais de coleta.

\begin{tabular}{lcc}
\hline Espécie & Código & Local de origem \\
\hline Trichogramma acacioi Brun, Moraes \& Soares & Trat 1 & Venda Nova, ES \\
Trichogramma atopovirilia Oatman \& Platner & Trat 2 & Sete Lagoas, MG \\
Trichogramma atopovirilia Oatman \& Platner & Trat 3 & Montes Claros, MG \\
Trichogramma brunni Nagaraja & Trat 4 & Alegre, ES \\
Trichogramma exiguum Pinto \& Platner & Trat 5 & Muniz Freire, ES \\
Trichogramma exigumm Pinto \& Platner & Trat 6 & Alegre, ES \\
Trichogramma pratissolii Querino \& Zucchi & Trat 7 & Venda Nova, ES \\
Trichogramma pretiosum Riley & Trat 8 & Conceição do Castelo, ES \\
Trichogramma pretiosum Riley & Trat 9 & Cristalina, GO \\
Trichogramma pretiosum Riley & Trat 10 & Paraopeba, MG \\
Trichogramma pretiosum Riley & Trat 11 & Pedra Preta, MT \\
Trichogramma pretiosum Riley & Trat 12 & Jaciara, MT \\
\hline
\end{tabular}


Fêmeas das espécies e/ou linhagens de Trichogramma, com até 24 horas de idade, foram individualizadas em tubos de vidro $(7,0 \times 1,3 \mathrm{~cm})$ tampados com filme plástico de PVC (Magipack®) e alimentadas com uma gotícula de mel puro. Vinte ovos de A. gemmatalis com, no máximo, 48 horas de desenvolvimento embrionário foram oferecidos por fêmea do parasitoide. O parasitismo foi permitido por 24 horas, após o qual as fêmeas foram retiradas dos tubos de vidro e as cartelas com os ovos, novamente, acondicionadas em outros tubos tampados com filme plástico de PVC.

Cada espécie e/ou linhagem (tratamento) foi representada por 15 repetições, com uma fêmea de um dos parasitoides por tubo. O número de ovos parasitados, percentual de emergência, o número de adultos emergidos por ovo, a razão sexual e o total de indivíduos por fêmea foram avaliados. Os dados foram submetidos à análise de variância e as médias comparadas pelo teste de Tukey $(\mathrm{P} \leq 0,05)$ com o programaSAS (SAS InstiTUTE, 1999). Os dados da razão sexual foram transformados em $\log (\overline{x+1})$ e a porcentagem de emergência em arc sen $\sqrt{x+1}$.

\section{RESULTADOS}

O parasitismo apresentado pela linhagem do Trat 2 foi o maior entre todas as linhagens testadas com 19 ovos parasitados de A. gemmatalis por fêmea. Essa linhagem teve desempenho 1,6 vezes maior que a do Trat 9, a qual se destacou, entre as demais, em segundo lugar e sem diferença significativa para as dos Trat 3, Trat 4 , Trat 5 e Trat $6(p<0,0001)$. O parasitismo das demais espécies e/ou linhagens de Trichogramma testadas variou de 6,8 a 8,10 ovos parasitados (Tabela 2).

A emergência dos descendentes de todas as espécies e/ou linhagens de Trichogramma em ovos de $A$. gemmatalis foi considerada elevada, tendo como destaque as dos Trat 8 e Trat 12 com 100\% de emergência, sendo que elas diferiram apenas do Trat 10 que apresentou a menor taxa com 89,70\% (p $=0,0227$ ) (Tabela 2).

A razão sexual foi o parâmetro que apresentou maior variação nos resultados $(p<0,0001)$. As linhagens dos Trat 7 e Trat 8 apresentaram uma razão sexual de 1,00, o que representa a emergência de $100 \%$ de indivíduos fêmea. Para as outras linhagens, as taxas de razão sexual também foram elevadas, no entanto, houve nascimento de machos, ficando entre 0,66 a 0,90 (Tabela 3 ).

Onúmero de adultos de Trichogramma, emergidos por ovo de A. gemmatalis, para as diferentes espécies e/ou linhagens, apresentou grande variação estatística ( $p<0,0001)$, no entanto, esta variação apresentada na análise não se refletiu nos números, sendo que os valores médios ficaram bem próximos variando de 1,53 a 2,35 para as Trat 7 e Trat 12, respectivamente (Tabela 3 ).

O total médio de fêmeas das diferentes espécies e/ ou linhagens Trichogramma emergidas mostra que a do Trat 2 foi a que melhor apresentou desempenho em ovos de A. gemmatalis, sendo confirmado pela análise estatística, pois a do Trat 6 que se destacou em segundo lugar neste parâmetro apresentou nascimento de 17,40 indivíduos fêmea contra 29,20 da Trat 2 ( $p<0,0001)$, sendo esta diferença significativa para um experimento que objetiva verificar desempenho de diferentes espécies/linhagens (Tabela 3).

Tabela 2 - Número de ovos parasitados e emergência (\%) de espécies e/ou linhagens de Trichogramma (Hymenoptera: Trichogrammatidae) em ovos de Anticarsia gemmatalis (Lepidoptera: Noctuidae). $25 \pm 1^{\circ} \mathrm{C}, 70 \pm 10 \%$ UR e fotoperíodo de 12 horas.

\begin{tabular}{|c|c|c|}
\hline Espécie/linhagem ${ }^{2}$ & № de ovos parasitados & Emergência $(\%)^{3}$ \\
\hline Trat 1 & $8,60 \pm 0,50 \quad \mathrm{CDE}$ & $95,40 \pm 1,61 \mathrm{AB}$ \\
\hline Trat 2 & $19,00 \pm 0,70 \mathrm{~A}$ & $97,90 \pm 1,02 \mathrm{AB}$ \\
\hline Trat 3 & $11,00 \pm 0,80 \quad B C$ & $98,50 \pm 0,82 \mathrm{AB}$ \\
\hline Trat 4 & $9,30 \pm 0,70$ & $97,70 \pm 1,17 \mathrm{AB}$ \\
\hline Trat 5 & $9,80 \pm 0,70$ & $94,00 \pm 1,64 \mathrm{AB}$ \\
\hline Trat 6 & $10,00 \pm 0,60$ & $97,50 \pm 1,38 \mathrm{AB}$ \\
\hline Trat 7 & $6,80 \pm 0,40$ & $98,50 \pm 1,05 \mathrm{AB}$ \\
\hline Trat 8 & $7,90 \pm 0,40$ & $100,00 \pm 0,00 \mathrm{~A}$ \\
\hline Trat 9 & $12,00 \pm 0,60$ & $97,70 \pm 1,15 \mathrm{AB}$ \\
\hline Trat 10 & $6,70 \pm 0,60$ & $89,70 \pm 6,59 \quad B$ \\
\hline Trat 11 & $8,70 \pm 0,80$ & $96,40 \pm 1,85 \mathrm{AB}$ \\
\hline Trat 12 & $8,10 \pm 0,60$ & $100,00 \pm 0,00 \mathrm{~A}$ \\
\hline $\mathrm{F}$ & 26,42 & 2,10 \\
\hline $\mathrm{P}$ & $<0,0001$ & 0,0227 \\
\hline
\end{tabular}

${ }^{1}$ Médias seguidas de mesma letra, por coluna, não diferem pelo teste de Tukey a $5 \%$ de probabilidade.

${ }^{2}$ Trat 1: linhagem de T. acacioi; Trat 2, Trat 3: linhagens de T. atopovirilia; Trat 4: linhagem de T. brunni; Trat 5, Trat 6: linhagens de T. exigumm; Trat 7: linhagem de T. pratissoli; Trat 8, Trat 9, Trat 10, Trat 11, Trat 12: linhagens de T. pretiosum. ${ }^{3}$ Dados transformados em arc sen $\sqrt{\mathrm{x}+1}$. 


\section{DISCUSSÃO}

As espécies e/ou linhagens testadas apresentaram grande variação em relação ao número de ovos parasitados, sendo que a linhagem do Trat 2 teve destaque no seu desempenho em parasitar ovos de A. gemmatalis. É esperado que estas variações ocorram principalmente por se tratar de indivíduos provenientes de diferentes regiões e por este motivo terem comportamento diferenciado, sendo que estas diferenças no potencial de parasitismo entre espécies e/ou linhagens têm sido relatadas (PrATISSOLI et al., 2008).

Além das características comportamentais intrínsecas, outros fatores podem estar relacionados ao potencial em parasitar decada espéciee/ou linhagem do parasitoide, sendo que entre estes destacam-se os fatores do próprio hospedeiro utilizado (Pratissoliet al., 2004b). Este fator é preponderante para que estes experimentos sejam realizados antes de se iniciar um programa de manejo fitossanitário onde se pretende utilizar parasitóides do gênero Trichogramma como uma das táticas de controle.

Dentre as características do ovo do hospedeiro que influi no parasitismo de espéciese/ou linhagens de Trichogramma estão o volume do ovo, espessura do córion, conteúdo nutricional, pois é conhecida a capacidade que estes parasitoides têm em reconhecer a qualidade do hospedeiro. Idade e forma de postura também podem afetar, além da taxa de parasitismo, a qualidade dos parasitoides emergidos (HOFFMANN et al., 2001; RoRIz, et al., 2006; RUKMOWATI-BROTODJOJO; WALTER, 2006).

Os fatores comportamentais de Trichogramma mostram também que, independente do hospedeiro utilizado, algumas espécies e/ou linhagens possuem maior capacidade em parasitar ovos do que outras devido à sua agressividade, mesmo sobre condições adversas (VIANNA et al., 2009), e isto confirma que $A$. gemmatalis é um excelente hospedeiro para Trichogramma e ainda apresenta grande potencial de ser controlado por esses parasitoides, pois todas as espécies/linhagens utilizadas parasitaram seus ovos e apresentaram descendentes.

Os ovos de A. gemmatalis demonstraram possuir características físico-químicas adequadas, que os tornam um hospedeiro em potencial para Trichogramma, pois todas as espécies e/ ou linhagens testadas apresentaram emergência superior a $94 \%$, exceto na do Trat 10 que, mesmo com emergência de $89,70 \%$, foi superior ao indicado como aceitável em programas de produção massal desse parasitoide (NAVA et al., 2007; Dias et al., 2008).

Tabela 3 - Razão sexual, número de adultos emergidos por ovo e total de fêmeas emergidas de espécies e/ou linhagens de Trichogramma (Hymenoptera: Trichogrammatidae) em ovos de Anticarsia gemmatalis (Lepidoptera: Noctuidae). $25 \pm$ $1^{\circ} \mathrm{C}, 70 \pm 10 \%$ UR e fotoperíodo de 12 horas

\begin{tabular}{|c|c|c|c|}
\hline Espécie/Linhagem² ${ }^{2}$ & Razão sexual $^{3}$ & № de adultos emergidos por ovo & $\begin{array}{l}\text { Total médio de fêmeas emergidas por } \\
\text { tratamento }\end{array}$ \\
\hline Trat 1 & $1,00 \pm 0,00 \mathrm{~A}$ & $1,92 \pm 0,12 \mathrm{ABC}$ & $15,40 \pm 0,88 \quad \mathrm{BCD}$ \\
\hline Trat 2 & $0,84 \pm 0,02 \quad \mathrm{BC}$ & $1,88 \pm 0,05 \mathrm{ABC}$ & $29,20 \pm 1,57 \mathrm{~A}$ \\
\hline Trat 3 & $0,76 \pm 0,03 \quad \mathrm{BC}$ & $1,90 \pm 0,09 \mathrm{ABC}$ & $16,30 \pm 1,61 \quad \mathrm{BCD}$ \\
\hline Trat 4 & $0,90 \pm 0,01 \quad \mathrm{~B}$ & $2,08 \pm 0,07 \mathrm{AB}$ & $17,00 \pm 1,34 \quad \mathrm{BC}$ \\
\hline Trat 5 & $0,87 \pm 0,02 \quad B$ & $2,08 \pm 0,11 \mathrm{AB}$ & $16,40 \pm 1,27 \quad B C D$ \\
\hline Trat 6 & $0,87 \pm 0,02 \quad B$ & $2,07 \pm 0,12 \mathrm{AB}$ & $17,40 \pm 1,12 \quad$ B \\
\hline Trat 7 & $1,00 \pm 0,00 \mathrm{~A}$ & $2,35 \pm 0,12 \mathrm{~A}$ & $15,50 \pm 1,03 \quad \mathrm{BCD}$ \\
\hline Trat 8 & $1,00 \pm 0,00 \mathrm{~A}$ & $1,71 \pm 0,08 \quad \mathrm{BC}$ & $13,70 \pm 1,02 \quad \mathrm{BCD}$ \\
\hline Trat 9 & $0,66 \pm 0,07 \quad C$ & $2,29 \pm 0,11 \mathrm{~A}$ & $16,60 \pm 1,71 \quad \mathrm{BCD}$ \\
\hline Trat 10 & $0,73 \pm 0,07 \quad \mathrm{BC}$ & $1,99 \pm 0,16 \mathrm{ABC}$ & $10,80 \pm 1,39 \quad \mathrm{D}$ \\
\hline Trat 11 & $0,79 \pm 0,06 \quad \mathrm{BC}$ & $2,25 \pm 0,14 \mathrm{~A}$ & $14,50 \pm 1,69 \quad \mathrm{BCD}$ \\
\hline Trat 12 & $0,89 \pm 0,03 \quad$ B & $1,53 \pm 0,09 \quad C$ & $10,90 \pm 0,91 \quad C D$ \\
\hline $\mathrm{F}$ & 16,95 & 4,74 & 12,37 \\
\hline $\mathrm{P}$ & $<0,0001$ & $<0,0001$ & $<0,0001$ \\
\hline
\end{tabular}

${ }^{1}$ Médias seguidas de mesma letra, por coluna, não diferem entre si pelo teste de Tukey, a 5\% de probabilidade.

${ }^{2}$ Trat 1: linhagem de T. acacioi; Trat 2, Trat 3: linhagens de T. atopovirilia; Trat 4: linhagem de T. brunni; Trat 5, Trat 6: linhagens de T. exigumm; Trat 7: linhagem de T.pratissoli; Trat 8, Trat 9, Trat 10, Trat 11, Trat 12: linhagens de T. pretiosum. ${ }^{3}$ Dados transformados em $\log (x+1)$. 
A razão sexual foi alta para a maioria das espécies e/ou linhagens utilizadas no experimento, sendo que o nascimento de uma maior quantidade de fêmeas é bastante desejável em programas de controle biológico, pois quanto maior o número de fêmeas maior o potencial de controle (CAÑETE; Foerster, 2003; Pratissoli et al., 2004b; WAKeIl et al., 2008). As taxas são consideradas altas com diferentes espécies de Trichogramma e hospedeiros (Pratissoli et al., 2004b; Pereira et al., 2004; Pratissoli et al., 2008), pois a razão sexual ideal deve ser superior a 0,5 , para criação massal de espécies de Trichogramma que representa a emergência de, pelo menos, um indivíduo fêmea por macho emergido (NAva et al., 2007; Dias et al., 2008). Esses resultados comprovam a qualidade do hospedeiro utilizado, pois a alocação sexual de fêmeas é, principalmente, devido à qualidade do hospedeiro e a competição (KAPRANAs et al., 2008).

Onúmero deadultos de Trichogramma, emergidos por ovo de A. gemmatalis, variou de1,53 a 2,35 (Tabela $3)$. O volume do ovo é o principal fator que influencia o número de parasitoides emergidos por ovo do hospedeiro, o que também influencia o tamanho do adulto de Trichogramma dependendo dos recursos nutricionais disponíveis para seu desenvolvimento (HondA; LuCK, 2001; NAvaet al., 2007; Diasetal., 2008). A variação no número de indivíduos emergidos por ovo do hospedeiro pode estar relacionada à capacidade de espécies e/ou linhagens do parasitoide apresentarem maior capacidade de reconhecer o ovo de A. gemmatalis (RoRIz et al., 2006; FACKNATH; WRIGHT, 2007), pois todas as espécies e/ou linhagens avaliadas eram mantidas em laboratório em ovos de A. kuehniella. Assim, A. gemmatalis tem potencial como hospedeiro de Trichogramma, no entanto, podemos observar que a linhagem do parasitoide também afeta o número de adultos emergidos por ovo desse parasitoide.

Ototal de fêmeas de Trichogramma spp. emergida por tratamento tem sido pouco utilizado para discutir trabalhos de comportamento de parasitismo desse parasitoide, porém, é um parâmetro que engloba todas as características desejáveis de um parasitoide tanto para sua criação massal como para a sua utilização no controle biológico de pragas. $\mathrm{O}$ maior total de fêmeas produzidas confirma o melhor desempenho do Trat 2, embora a do Trat 9 seja proveniente de ovos de $A$. gemmatalis em soja. Isto mostra que espécies não coletadas no hospedeiro de origem podem apresentar potencial de controle para determinada espécie. Além disso, diferenças para as outras espécies/linhagens podem estar relacionadas ao fato desses parasitoides terem passado gerações sucessivas em laboratório em ovos de A. kuehniella (Pratissoli et al., 2004b; Dias et al., 2008). A linhagem do Trat 2 apresentou melhores resultados, o que mostra que, apesar dessa linhagem não ter sido coletada em ovos do hospedeiro estudado e o fato dela ter passado por sucessivas gerações em ovos de $A$. kuehniella, não afetou sua capacidade de reconhecer outros hospedeiros. Isto mostra que outras linhagens podem apresentar alta capacidade de reprodução em um hospedeiro de qualidade independente de sua origem (REITDORF; STEIDLE, 2002; CHOW et al., 2005; FACKNATH; WRIGHT, 2007).

T. atopovirilia (linhagem do Trat 2) foi coletada na cultura do milho e, mesmo assim, apresentou potencial para o controle de A. gemmatalis, isto se deve também ao fato de ter relatos dessa espécie ocorrendo naturalmente, parasitando ovos dessa praga (CAÑete; Foerster, 2003). No entanto, a linhagem do Trat 3 também é da espécie T. atopovirilia e ela apresentou resultados inferiores aos apresentados para a do Trat 2, o que mostra a importância de se estudar o comportamento de diferentes espécies e linhagens antes de se iniciar um programa de controle biológico com Trichogramma.

Apesar do fato da linhagem do Trat 2 ter apresentado melhor desempenho em ovos de A. gemmatalis, as outras linhagens de Trichogramma também apresentaram resultados promissores para o controle dessa praga. Essas variações entre espécies de Trichogramma, principalmente no parasitismo, mostram a importância de seavaliar o maior número de espécies e linhagens desse parasitoide.

\section{CONCLUSÕES}

A linhagem do Trat 2 apresentou melhor desempenho em ovos de A. gemmatalis. Anticarsia gemmatalis tem potencial de ser controlada por espécies de Trichogramma.

\section{REFERÊNCIAS}

CAÑETE, C.L.; FOERSTER, L.A. Incidência natural e biologia de Trichogramma atopovirilia Oatman \& Platner, 1983 (Hymenoptera, Trichogrammatidae) em ovos de Anticarsia gemmatalis Hübner, 1818 (Lepidoptera, Noctuidae). Revista Brasileira de Entomologia, v.47, n.2, p.201-204, 2003.

CHOW, J.K.; AKHTAR, Y.; ISMAN, M.B. The effects of larval experience with a complex plant latex on subsequent feeding and oviposition by the cabbage looper moth: Trichoplusia ni (Lepidoptera: Noctuidae). Chemoecology, v.15, n.3, p.129-133, 2005.

DIAS, N.S.; PARRA, J.R.P.; LIMA, T.C.C. Seleção de hospedeiro alternativo para três espécies de tricogramatídeos neotropicais. Pesquisa Agropecuária Brasileira, v.43, n.11, p.1467-1473, 2008. 
FACKNATH, S.; WRIGHT, D.J. Is host selection in leafminer adults influenced by pre-imaginal or early adult experience? Journal of Applied Entomology, v.131, n.8, p.505-512, 2007.

GREENE, G.L.; LEPPLA, N.C.; DICKERSON, W.A. Velvetbean caterpillar: a rearing procedure and artificial medium. Journal of Economic Entomology, v.69, n.4, p.487488,1976

GRIESHOP, M.J.; FLINN, P.W.; NECHOLS, J.R.; CAMPBELL, J.F. Effects of fine-grain habitat complexity on egg parasitism by three species of Trichogramma. Biological Control, v.45, n.3, p.328-336, 2008.

HEGAZI, E.; HERZ, A.; HASSAN, S.A.; KHAFAGI, W.E.; AGAMY, E.; ZAITUN, A.; EL-AZIZ, G.A.; SHOWEIL, S.; EL-SAID, S.; KHAMIS, N. Field efficiency of indigenous egg parasitoids (Hymenoptera, Trichogrammatidae) to control the olive moth (Prays oleae, Lepidoptera, Yponomeutidae) and the jasmine moth (Palpita unionalis, Lepidoptera, Pyralidae) in an olive plantation in Egypt. Biological Control, v.43, n.2, p.171187, 2007.

HOFFMANN, M.P.; ODE, P.R.; WALKER, D.L.; GARDNER, J.; VAN NOUHUYS, S.; SHELTON, A.M. Performance of Trichogramma ostriniae (Hymenoptera: Trichogrammatidae) reared on factitious hosts, including the target host, Ostrinia nubilalis (Lepidoptera: Crambidae). Biological Control, v.21, p.1-10, 2001.

HONDA, J.Y.; LUCK, R.F. Interactions between host attributes and wasp size: a laboratory evaluation of Trichogramma platneri as an augmentative biological control agent for two avocado pests. Entomologia Experimentalis et Applicata, v.100, n.1, p.1-13, 2001.

HUMMEL, R.L.; WALGENBACH, J.F.; HOYT, G.D.; KENNEDY, G.G. Effects of production system on vegetable arthropods and their natural enemies. Agricultural Ecosystems and Environment, v.93, n.1-3, p.1-12, 2002.

KAPRANAS, A.; PACHECO, P.; FORSTER, L.D.; MORSE, J.G.; LUCK, R.F. Precise sex ratios manifested by several encyrtid parasitoids (Hymenoptera: Encyrtidae) of brown soft scale, Coccus hesperidum L. (Hemiptera: Coccidae). Behavioral Ecology of Sociobiology, v.62, n.6, p.901-912, 2008.

LOURENÇÃO, A.L.; PEREIRA, J.C.V.N.A.; MIRANDA, M.A.C.; AMBROSANO, G.M.B. Avaliação dos danos causados por percevejos e por lagartas em genótipos de soja de ciclos precoce e semiprecoce. Pesquisa Agropecuária Brasileira, v.35, n.5, p.879-886, 2000.

MACRAE, T.C.; BAUR, M.E.; BOETHEL, D.J.; FITZPATRICK, B.J.; GAO, A.G.; GAMUNDI, J.C.; HARRISON, L.A.; KABUYE, V.T.; MCPHERSON, R.M.; MIKLOS, J.A.; PARADISE, M.S.; TOEDEBUSCH, A.S.; VIEGAS, A. Laboratory and field evaluations of transgenic soybean exhibiting high-dose expression of a synthetic Bacillus thuringiensis cry1A gene for control of Lepidoptera. Journal of Economic Entomology, v.98, n.2, p.577-587, 2005.

MATOS NETO, F.C.; ZANUNCIO, J.C.; PICANÇO, M.C.; CRUZ, I. Reproductive characteristics of the predator Podisus nigrispinus fed with an insect resistent soybean variety. Pesquisa Agropecuária Brasileira, v.37, n.7, 917-924, 2002.

MIKLOS, J.A.; ALIBHAI, M.F.; BLEDIG, S.A.; CONNORWARD, D.C.; GAO, A.G.; HOLMES, B.A.; KOLACZ, K.H.; KABUYE, V.T.; MACRAE, T.C.; PARADISE, M.S.; TOEDEBUSCH, A.S.; HARRISON, L.A. Characterization of soybean exhibiting high expression of a synthetic Bacillus thuringiensis cry1A transgene that confers a high degree of resistance to Lepidopteran pests. Crop Science, v.47, p.148-157, 2007

NAVA, D.E.; TAKAHASHI, K.M.; PARRA, J.R.P. Linhagens de Trichogramma e Trichogrammatoidea para o controle de Stenoma catenifer. Pesquisa Agropecuária Brasileira, v.42, n.1, p.9-16, 2007.

OLIVEIRA, H.N.; ZANUNCIO, J.C.; PRATISSOLI, D.; CRUZ, I. Parasitism rate and viability of Trichogramma maxacalii (Hym.: Trichogrammatidae) parasitoid of the Eucalyptus defoliator Euselasia apisaon (Lep.: Riodinidae), on eggs of Anagasta kuehniella (Lep.: Pyralidae). Forest Ecology and Management, v.130, n.1, p.1-6, 2000.

OLIVEIRA, H.N. DE; PRATISSOLI, D.; ZANUNCIO, J.C.; SERRÃO, J.E. Influência da idade de ovos de Oxydia vesulia no parasitismo de Trichogramma maxacalii. Pesquisa Agropecuária Brasileira, v.38, n.4, p.551-554, 2003.

PEREIRA, F.F.; BARROS, R.; PRATISSOLI, D.; PARRA, J.R.P. Biologia e exigências térmicas de Trichogramma pretiosum Riley e T. exiguum Pinto \& Platner (Hymenoptera: Trichogrammatidae) criados em ovos de Plutella xylostella (L.) (Lepidoptera: Plutellidae). Neotropical Entomology, v.33, n.2, p.231-236, 2004.

PRATISSOLI, D.; FORNAZIER, M.J.; HOLTZ, A.M.; GONÇALVES, J.R.; CHIORAMITAL, A.B.; ZAGO, H. Ocorrência de Trichogramma pretiosum em áreas comerciais de tomate, no Espírito Santo, em regiões de diferentes altitudes. Horticultura Brasileira, v.21, n,1, p.73-76, 2002.

PRATISSOLI, D.; ZANUNCIO, J.C.; VIANNA, U.R.; ANDRADE, J.S.; GUIMARÃES, E.M.; ESPINDULA, M.C. Fertility life table of Trichogramma pretiosum and Trichogramma acacioi on eggs of Anagasta kuehniella at different temperatures. Pesquisa Agropecuária Brasileira, v.39, n.2, p.193-196, 2004a.

PRATISSOLI, D.; OLIVEIRA, H.N.; GONÇALVES, J.R.; ZANUNCIO, J.C.; HOLTZ, A.M. Changes in biological characteristics of Trichogramma pretiosum (Hym. Trichogrammatidae) reared on eggs of Anagasta 
kuehniella (Lep.: Pyralidae) for 23 generations. Biocontrol Science and Technology, v.14, n.3, p.313-319, 2004 b.

PRATISSOLI, D.; POLANCZYK, R.A.; HOLTZ, A.M.; DALVI, L.P.; SILVA, A.F.; SILVA, L.N. Selection of Trichogramma species for controlling the diamondback moth. Horticultura Brasileira, v.26, n.2, p.259-261, 2008.

REITDORF, K.; STEIDLE, J.L.M. Was Hopkins right? Influence of larval and early adult experience on the olfactory response in the granary weevil Sitophilus granarius (Coleoptera, Curculionidae). Physiological Entomology, v.27, n.2, p.223-227, 2002.

RORIZ, V.; OLIVEIRA, L.; GARCIA, P. Host suitability and preference studies of Trichogramma cordubensis (Hymenoptera: Trichogrammatidae). Biological Control, v.36, n.3, p.331-336, 2006

RUKMOWATI-BROTODJOJO, R.R.; WALTER, G.H. Oviposition and reproductive performance of a generalist parasitoid (Trichogramma pretiosum) exposed to host species that differ in their physical characteristics. Biological Control, v.39, n.3, p.300-312, 2006.

SAS INSTITUTE. “SAS/STAT 1999” User's guide: statistics, version 8, v.2, SAS Institute Inc., Cary, NC, USA. 1999.

SHARLEY, D.J.; HOFFMANN, A.A.; THOMSON, L.J. The effects of soil tillage on beneficial invertebrates within the vineyard. Agricultural and Forest Entomology, v.10, n.3, p.233-243, 2008.
SOARES, M.A.; LEITE, G.L.D.; ZANUNCIO, J.C.; ROCHA, S.L.; DE SÁ, V.G.M.; SERRÃO, J.E. Flight capacity, parasitism and emergence of five Trichogramma (Hymenoptera: Trichogrammatidae) species from forest areas in Brazil. Phytoparasitica, v.35, n.2, p.314-318, 2007.

THOMAS, J.D.; BOETHEL, D.J. Synergism of insecticides in tests with resistant soybean looper larvae (Lepidoptera: Noctuidae) in the laboratory and field. Journal of Economic Entomology, v.87, n.6, p.1416-1422, 1994.

VIANNA, U.R.; PRATISSOLI, D.; ZANUNCIO, J.C.; LIMA, E.R.; BRUNNER, J.; PEREIRA, F.F.; SERRÃO, J.E. Insecticide toxicity to Trichogramma pretiosum (Hymenoptera: Trichogrammatidae) females and effect on descendant generation. Ecotoxicology, v.18, n.2, p.180-186, 2009.

WAKEIL, N.E.; FARGHALY, H.T.; RAGAB, Z.A. Efficacy of inundative releases of Trichogramma evanescens in controlling Lobesia botrana in vineyards in Egypt. Journal of Pesticide Science, v.81, n.1, p.49-55, 2008.

WALKER, D.R.; ALL, J.N.; MCPHERSON, R.M.; BOERMA, H.R.; PARROTT, W.A. Field evaluation of soybean engineered with a synthetic cry $1 A c$ transgene for resistance to corn earworm, soybean looper, velvetbean caterpillar (Lepidoptera: Noctuidae), and lesser cornstalk borer (Lepidoptera: Pyralidae). Journal of Economic Entomology, v.93, n.3, p.613-622, 2000.

Recebido em $1 / 2 / 10$

Aceito em 9/1/11 